\title{
Statistical Analysis of Risk Factors for Cardiovascular Disease in Malakand Division
}

\author{
Salahuddin \\ Department of Statistics, University of Peshawar, Pakistan \\ Fazle Rabbi \\ GHSS, Baidara, Swat, Pakistan \\ Fazli Qadir \\ Department of Statistics, University of Peshawar, Pakistan \\ Mehnaz Khattak \\ Department of Statistics, Jinnah College for Women \\ University of Peshawar, Pakistan
}

\begin{abstract}
Several studies have been conducted to investigate the incidence of Myocardial Infarction (MI) and to determine the possible risk factors for the disease. In this study, a statistical analysis has been performed to look at the association of $\mathrm{Ml}$ with various risk factors such as diabetes, cholesterol, hypertension, sex, smoking, obesity, family history and age in Malakand division. A total of 700 patients were examined and their personal and medical data were collected. For each patient, the phenomenon $\mathrm{MI}$ was studied in relation to different risk factors. The analyses suggest that hypertension, smoking, diabetes, cholesterol level and family history were important risk factors for the occurrence of myocardial infarction.
\end{abstract}

\section{Introduction}

Cardiovascular disease has emerged as one of the major health problem in Pakistan. Every year in Pakistan, large number of people die due to this disease. There are many types of cardiovascular diseases. One of these diseases is the myocardial infarction (MI). Large number of people die every year due to MI. A heart attack or myocardial infarction occurs when a coronary artery abruptly fails to deliver blood to a part of our heart.

Various studies have been conducted to investigate the incidence of $\mathrm{MI}$ and to determine the possible risk factors for the disease (for example see, Ruiswyk. et. al. (1993); Flack. et. al. (1995); Rabajoli. et. al. (1996); Capewell. et. al. (1996); Aminian. et. al. (1998); Tariq et. al. (1998)). They all studied various demographic and medical risk factors of MI.

Another study was conducted by Ahmad et. al. (1995) regarding 108 patients with acute MI. They found that important risk factors prevalent in the study were smoking, lack of exercise, stress, hypertension, diabetes mellitus and disturbed lipid levels.

Pais et. al. (2001) studied the increased risk of acute myocardial infarction with beedi and cigarette smoking in India. They conducted their study in a teaching 
hospital in Banglore. They found that important risk factor prevalent in the study was smoking.

The effects of various risk factors on MI in Malakand Division, has been carried out by using the data obtained from the cardiology unit Saidu group of teaching hospitals, Saidu Sharif, Swat. The associations of various risk factors with the occurrence of Ml were determined through the odds ratios.

\section{Methodology}

A total of 700 patients (512 males and 188 females) were examined from the cardiology unit of Saidu group of teaching hospitals, Saidu Sharif, Swat for MI to investigate the risk factors of MI like diabetes, cholesterol, hypertension, $\operatorname{sex}(\operatorname{sex}=0$, if patient is female and sex $=1$, if patient is male), smoking, obesity, family history (that is family history of $\mathrm{MI}$ ) and age. The odd ratios have been computed to see the significant factors associated with MI.

\section{Odds and the Odds Ratio}

It is sometimes helpful to describe the chance that a binary response variable leads to a success in terms of the odds of that event. When two sets of binary data are to be compared, a relative measure of the odds of success in one set relative to that in the other is referred to as the odds ratio.

Suppose a $(2 \times 2)$ table is given as:

\begin{tabular}{|c|c|c|}
\hline & $\begin{array}{c}\text { Number of } \\
\text { Success }\end{array}$ & $\begin{array}{c}\text { Number of } \\
\text { failures }\end{array}$ \\
\hline Data set 1 & $a$ & $b$ \\
Data set 2 & $c$ & $d$ \\
\hline
\end{tabular}

Then the odds ratio is given as:

$$
\hat{\psi}=\frac{\hat{p}_{1} /\left(1-\hat{p}_{1}\right)}{\hat{p}_{2} /\left(1-\hat{p}_{2}\right)}=\frac{a d}{b c}
$$

This estimate is the ratio of the products of the two pairs of diagonal elements in the above $2 \times 2$ table, and for this reason, $\hat{\psi}$ is sometimes referred to as the cross- product ratio.

The association between two factors can be tested via odds ratio. The null hypothesis to be tested in this case is:

$$
H_{0}: \psi=1 \text { or equivalently, } H_{0}: \ln (\psi)=0
$$

Note that testing above hypothesis is same as testing $H_{0}: \beta_{1}=0$ in simple logistic model: 


$$
\ln \left(\frac{p}{1-p}\right)=\beta_{0}+\beta_{1} x
$$

The null hypothesis $H_{0}: \psi=1$ or $\ln (\psi)=0$ may be tested using the test statistic

$$
Z=\frac{\ln (\hat{\psi})}{S . E\{\ln (\hat{\psi})\}},
$$

which has an approximate standard normal distribution.

An approximate $100(1-\alpha) \%$ confidence interval for $\ln (\psi)$ is constructed as:

$$
\ln (\hat{\psi}) \pm Z_{\alpha / 2} S . E\{\ln (\hat{\psi})\}
$$

For example, a $95 \%$ confidence interval for $\ln (\psi)$ is given by

$$
\ln (\hat{\psi}) \pm 1.96 S . E\{\ln (\hat{\psi})\}
$$

The confidence interval given by equation (1) on inversion will give us the confidence interval for $\psi$ as

$$
\hat{\psi} e^{-z} \alpha / 2^{S . E .}<\psi<\hat{\psi} e^{-z} \alpha / 2^{S . . E .}
$$

If the interval contains unity, it indicates independence; otherwise an association is indicated.

\section{Odds Ratio Analyses and Interpretation}

\section{1) Myocardial infarction versus sex:}

Consider the following table:

Table 1: Myocardial infarction versus sex

\begin{tabular}{|c|c|c|c|}
\hline \multirow{2}{*}{ Sex } & \multicolumn{2}{|c|}{ MI } & \multirow{2}{*}{ Total } \\
\cline { 2 - 3 } & 0 & 1 & \\
\hline 0 & 56 & 132 & 188 \\
\hline 1 & 144 & 368 & 512 \\
\hline Total & 200 & 500 & 700 \\
\hline
\end{tabular}

In this case the estimated odds of having $\mathrm{Ml}$ are 1.084 times more for males as compared to females. Hence the males are more likely to have MI than females. The estimated log odds ratio is 0.081 and its asymptotic standard error is 0.187 , which is highly significant at $1 \%$ level of significance. A $95 \%$ confidence interval for the true log odds ratio is $(-0.286,0.448)$ and so the $95 \%$ confidence interval for the true odds ratio is $(0.751,1.564)$. The confidence interval shows a significant relationship between sex and myocardial infarction. Hence we can conclude that myocardial infarction is associated with sex. 


\subsection{Myocardial infarction versus smoking:}

Consider the following table:

Table 2: Myocardial infarction versus smoking

\begin{tabular}{|c|c|c|c|}
\hline \multirow{2}{*}{ Smoking } & \multicolumn{2}{|c|}{ MI } & \multirow{2}{*}{ Total } \\
\cline { 2 - 3 } & 0 & 1 & \\
\hline 0 & 168 & 357 & 525 \\
\hline 1 & 32 & 143 & 175 \\
\hline Total & 200 & 500 & 700 \\
\hline
\end{tabular}

In this case the estimated odds of having myocardial infarction are 2.103 times more for smokers as compared to non-smokers. Hence the smokers are more likely to have myocardial infarction than non-smokers. The estimated log odds ratio is 0.743 and its asymptotic standard error is 0.217 , which is highly significant at $1 \%$ level of significance. A 95\% confidence interval for the true log odds ratio is $(0.318,1.168)$ and hence the $95 \%$ confidence interval for the true odds ratio is $(1.374,3.218)$. The confidence interval shows a significant relationship between smoking and myocardial infarction. Hence we can conclude that myocardial infarction is associated with smoking.

\subsection{Myocardial infarction versus diabetes:}

Consider the following table:

Table 3: Myocardial infarction versus diabetes

\begin{tabular}{|c|c|c|c|}
\hline \multirow{2}{*}{ Diabetes } & \multicolumn{2}{|c|}{ MI } & \multirow{2}{*}{ Total } \\
\cline { 2 - 3 } & 0 & 1 & \\
\hline 0 & 193 & 357 & 550 \\
\hline 1 & 7 & 143 & 150 \\
\hline Total & 200 & 500 & 700 \\
\hline
\end{tabular}

In this case the estimated odds of having myocardial infarction are 11.044 times more for diabetic as compared to non-diabetic. Hence the persons with diabetes are more likely to have myocardial infarction than those who do not have diabetes. The estimated log odds ratio is 2.402 and its asymptotic standard error is 0.397 , which is highly significant at $1 \%$ level of significance. A $95 \%$ confidence interval for the true log odds ratio is $(1.624,3.180)$ and hence the $95 \%$ confidence interval for the true odds ratio is $(5.072,24.047)$. The confidence interval shows a significant relationship between diabetes and myocardial infarction. Hence we can conclude that myocardial infarction is associated with diabetes. 
4.4 Myocardial infarction versus family history of myocardial infarction:

Consider the following table.

Table 4: Myocardial infarction versus family history of myocardial infarction

\begin{tabular}{|c|c|c|c|}
\hline \multirow{2}{*}{$\begin{array}{c}\text { Family } \\
\text { History }\end{array}$} & \multicolumn{2}{|c|}{ MI } & \multirow{2}{*}{ Total } \\
\cline { 2 - 3 } & 0 & 1 & \\
\hline 0 & 174 & 336 & 510 \\
\hline 1 & 26 & 164 & 190 \\
\hline Total & 200 & 500 & 700 \\
\hline
\end{tabular}

In this case the estimated odds of having myocardial infarction are 3.266 times more for those who have a family history of myocardial infarction as compared to those who do not have family history of myocardial infarction. Hence the persons with a family history of myocardial infarction are more likely to have myocardial infarction than those who do not have family history of myocardial infarction. The estimated log odds ratio is 1.184 and its asymptotic standard error is 0.231 , which is highly significant at $1 \%$ level of significance. A $95 \%$ confidence interval for the true log odds ratio is $(0.731,1.637)$ and hence the $95 \%$ confidence interval for the true odds ratio is $(2.077,5.136)$. The confidence interval shows a significant relationship between family history and myocardial infarction. Hence we can conclude that myocardial infarction is associated with family history of myocardial infarction.

\subsection{Myocardial infarction versus cholesterol:}

Consider the following table:

Table 5: Myocardial infarction versus cholesterol

\begin{tabular}{|c|c|c|c|}
\hline \multirow{2}{*}{ Cholesterol } & \multicolumn{2}{|c|}{ MI } & \multirow{2}{*}{ Total } \\
\cline { 2 - 3 } & 0 & 1 & \\
\hline 0 & 187 & 390 & 577 \\
\hline 1 & 13 & 110 & 123 \\
\hline Total & 200 & 500 & 700 \\
\hline
\end{tabular}

Here the estimated odds of having myocardial infarction are 4.057 times more for those who have a high cholesterol level as compared to those having low cholesterol level. Hence the persons with a high cholesterol level are more likely to have myocardial infarction than those who have a low cholesterol level. The estimated log odds ratio is 1.400 and its asymptotic standard error is 0.306 , which is highly significant at $1 \%$ level of significance. A $95 \%$ confidence interval for the true log odds ratio is $(0.800,1.999)$ and hence a $95 \%$ confidence interval for the true odds ratio is $(2.227,7.391)$. The confidence interval shows a 
significant relationship between high cholesterol level and myocardial infarction. Hence we can conclude that myocardial infarction is associated with high cholesterol level.

\subsection{Myocardial infarction versus hypertension:}

Consider the following table.

Table 6: Myocardial infarction versus hypertension

\begin{tabular}{|c|c|c|c|}
\hline \multirow{2}{*}{ Hypertension } & \multicolumn{2}{|c|}{ MI } & \multirow{2}{*}{ Total } \\
\cline { 2 - 3 } & 0 & 1 & \\
\hline 0 & 174 & 286 & 460 \\
\hline 1 & 26 & 214 & 240 \\
\hline Total & 200 & 500 & 700 \\
\hline
\end{tabular}

In this case the estimated odds of having myocardial infarction are 5.008 times more for persons having hypertension as compared to those who do not have hypertension. Hence the persons with hypertension are more likely to have myocardial infarction than those who do not have hypertension. The estimated log odds ratio is 1.611 and its asymptotic standard error 0.229 , which is highly significant at $1 \%$ level of significance. A $95 \%$ confidence interval for the true log odds ratio is $(1.162,2.060)$ and hence the $95 \%$ confidence interval for the true odds ratio is $(3.197,7.845)$. The confidence interval shows a significant relationship between the hypertension and myocardial infarction. Hence we can conclude that myocardial infarction is associated with the presence of hypertension.

\subsection{Myocardial infarction versus obesity:}

Consider the following table:

Table 7: Myocardial infarction versus obesity

\begin{tabular}{|c|c|c|c|}
\hline \multirow{2}{*}{ Obesity } & \multicolumn{2}{|c|}{ MI } & \multirow{2}{*}{ Total } \\
\cline { 2 - 3 } & 0 & 1 & \\
\hline 0 & 184 & 427 & 611 \\
\hline 1 & 16 & 73 & 89 \\
\hline Total & 200 & 500 & 700 \\
\hline
\end{tabular}

In this case the estimated odds of having myocardial infarction are 1.966 times more for persons having obesity than those who have no obesity. Hence the persons having obesity are more likely to have myocardial infarction than those who have no obesity. The estimated log odds ratio is 0.676 and its asymptotic standard error is 0.290 , which is highly significant at $1 \%$ level of significance. A $95 \%$ confidence interval for the true log odds ratio is $(0.108,1.244)$ and hence 
the $95 \%$ confidence interval for the true odds ratio is $(1.114,3.471)$. The confidence interval shows a significant relationship between obesity and myocardial infarction. Hence we can conclude that myocardial infarction is associated with obesity.

\subsection{Myocardial Infarction versus Age ( $>$ or $=60$ years):}

Consider the following table.

\section{Table 8: Myocardial infarction versus age ( $>$ or $=60$ years)}

\begin{tabular}{|c|c|c|c|}
\hline \multirow{2}{*}{ Age } & \multicolumn{2}{|c|}{ MI } & \multirow{2}{*}{ Total } \\
\cline { 2 - 3 } & 0 & 1 & \\
\hline 0 & 52 & 160 & 212 \\
\hline 1 & 148 & 340 & 488 \\
\hline Total & 200 & 500 & 700 \\
\hline
\end{tabular}

In this case the estimated odds of having myocardial infarction are 0.747 times more for persons having age less than 60 years than those having age equal to or greater than 60 years. Hence the persons having age equal to or greater than 60 years are more likely to have myocardial infarction than those who have age less than 60 years. The estimated log odds ratio is -0.292 and its asymptotic standard error is 0.188 , which is highly significant at $5 \%$ level of significance. A $95 \%$ confidence interval for the true log odds ratio is $(-0.660,0.076)$ and hence the $95 \%$ confidence interval for the true odds ratio is $(0.517,1.079)$. The confidence interval shows a significant relationship between age and myocardial infarction. Hence we can conclude that myocardial infarction is associated with age (equal to or greater than 60 years).

\section{Conclusion}

The main objective of present study was to determine most likely risk factors of $\mathrm{MI}$ and to model the incidence of $\mathrm{Ml}$ in patients arriving at cardiology unit Saidu group of teaching hospitals Saidu Sharif Swat. In this study 700 patients were examined and their personal and medical data were taken. For each patient, the phenomena of $\mathrm{Ml}$ was studied in relation to different risk factors, like hypertension, cholesterol, diabetes, smoking, sex, obesity, age and family history of MI.

Odds ratio analyses were performed to look at the association of MI with different risk factors of MI. The odds of $\mathrm{Ml}$ for male patients were 1.084 times more than for female patients. This shows an association between Ml and sex. The odds of MI for smoker patients were 2.103 times more than for non-smokers. This indicates an association between $\mathrm{Ml}$ and smoking. The odds of $\mathrm{Ml}$ for diabetics were 11.044 times more than for non-diabetics. This shows a significant relationship between $\mathrm{MI}$ and diabetes. The odds of $\mathrm{Ml}$ for patients having family history of Ml were 3.266 times more than for those having no family history of Ml. 
This also shows a significant association between $\mathrm{Ml}$ and family history of $\mathrm{Ml}$. There was a significant association between $\mathrm{Ml}$ and high cholesterol level. The odds of Ml for high cholesterol were 4.057 times more than those patients having low cholesterol level. The odds of MI for hypertensive patients were 5.008 times more than for non-hypertensive patients. This also indicates a significant association between $\mathrm{Ml}$ and hypertension. There was also an association between obesity and MI. The odds of MI for those patients who had obesity were 1.966 times more than for those who had no obesity. Age and Ml also proved to be associated with each other. The odds of $\mathrm{Ml}$ for patients of age less than 60 years were 0.747 times more than for those of age equal to or greater than 60 years.

In summary, we conclude from the analysis of random sample of 700 patients that $\mathrm{MI}$ has a strong association with hypertension, cholesterol, diabetes, smoking, sex, obesity, age and family history of myocardial infarction.

\section{References}

1. Aminian, B., Habibzadeh, F. and Moarref, A.R. (1998). "Factor analysis of cardiogenic shock complicating acute myocardial infarction". National Iranian Oil Company out patient polyclinics, Shiraz, Iran.

2. Capewell, S., Kendrick, S., Boyd, J., Cohen, G., Juszczak, E. and Clarke, J. (1996). "Measuring outcomes: one month survival after acute myocardial infarction in Scotland". British medical Journal, 76, pp: 70-75.

3. Flack, J.M., Neatson, J., Grimm, R., Shih, J., Cutler, J., Ensrud, K. and MacMahon, S. (1995). "Blood pressure and mortality Among Men with prior Myocardial Infarction". Journal of American Heart Association, 92, pp: 2437-2445.

4. Tariq et. at. (1998). Delay in Presentation in Patients with Acute Myocardial Infarction. Ann King Edward Med College, 4(4):33-7.

5. Ahmed, N., Mirza, T. and Malik, N. (1995). Risk factors in acute Myocardial Infarction. Journal of Pakistan Institute of Medical Sciences, Islamabad, 6(1,2): 352-5.

6. Pais, P., Fay, M.P. and Yusuf, S. (2001). "Increased Risk of Myocardial Infarction Associated With Beedi and Cigarette Smoking in India". Indian Heart Journal; 53: pp: 731-735.

7. Rabajoli, F., Arneodo, D., Balzola, F., Leo, L. and Vineis, P. (1996). "Moderate alcohol intake and risk of myocardial infarction among nonsmokers". European journal of Public Health, 6(3), pp: 227-230.

8. Ruiswyk, J.V., Hartz, A., Kuhun, E., Krakuer, H., Young, M. and Rimm, A. (1993). "A measure of mortality risk for elderly patients with acute myocardial infarction". Division of General Internal Medicine, Medical college of Wisconsin, Milwaukee.13 (2), pp: 152-160. 